\title{
State of knowledge of the tachinid fauna of Eastern Asia, with new data from North Korea. Part II. Tachininae
}

\author{
Agnieszka DRABER-MOŃKO \\ Museum and Institute of Zoology,PAS, Wilcza 64,00-679Warszawa; e-mail: drabber@miiz.waw.pl
}

\begin{abstract}
The present paper is a continuation of an earlier paper concerned with the Tachinidae collected in North Korea by five expeditions of researchers from the Institute of Zoology PAS, Warsaw, Poland and is specifically concerned with the second part of the collections - the subfamily Tachininae. Twenty nine species representing 15 genera were identified in the material. Twenty four species are reported for the first time in the fauna of Korea. Among these species were 3 very rare, known only from original descriptions: Nigara gracilis Richter, Peleteria pallida Zimin and Tachina (Tachina) majae (Zimin). Ten rarely registered species are illustrated.
\end{abstract}

Key words: Diptera, Tachinidae, Tachininae, North Korea

\section{INTRODUCTION}

The first part of this paper (Draber-Mońko 2008) was concerned with the Tachinidae belonging to the subfamily Phasiinae. Thirteen species of the phasiine flies were identified and most of them had been previously unknown from the territory of Korea. Two new species are described and illustrated: Dionaea karinae Draber-Mońko and Hemvda dominikae DraberMońko. The first part also contains a discussion of the present state of knowledge of the Tachinidae fauna of the Far East and an exhaustive list of relevant papers concerned with this region.

The present paper is devoted to Tachinidae species belonging to the subfamily Tachininae. The Tachininae are a large subfamily with about 550 species recorded from the Palaearctic Region (Herting \& Dely-Draskovits 1993, Ziegler \& Shima 1996), about 250 species from the Russian Far East (Ziegler \& Shima 1996. Richter 2004), more than 135 species from Japan (Richter 2004. Tachi \& Shima 2002, 2005, 2006), about 330 species from China (O'Hara, Shima \& Zhang 2009), about 420 species from the Nearctic Region (O'Hara \& Wood 2004) and about 260 species from the Oriental Region (Crosskey 1977).

Twenty nine species belonging to 15 genera of the tachinine flies were identified and twenty four of them had been previously unknown from entire Korea.

The species of the subfamily recorded from Korea by previous authors are also mentioned in this paper. The authors are: Han \& Kim 1983. Herting \& Dely-Draskovits 1993. Hua 2006. Lim \& Han 2008. O'Hara. Shima \& Zhang 2009. Richter 1988, 2004. Shima 1992. Shima. Han \& Tachi 2010. Tachi \& Shima 1998, 2000, 2002, 2006. Ziegler \& Shima 1996. Zimin 1980. Zimin \& Kolomiets 1984.

Nowadays. 53 species belonging to 21 genera of tachinine flies are known from entire Korea.

The majority of the tachinine species are endoparasitoids mostly of caterpillars of Lepidoptera belonging to many different families. A few are endoparasitoids of Coleoptera of the Chrysomelid and Scarabaeid families. and Hymenoptera of the Eumenid. Pamphiliid and 
Tenthredinid families. One species known to develop in Chilopoda of the family Lithobiidae. Dermaptera of the family Forficulidae. Diptera of the family Tipulidae and Orthoptera of the family Tettigoniidae.

\section{MATERIAL AND METHODS}

The material collected in North Korea by researchers from the Institute of Zoology PAS is fairly small. However, because of poor knowledge of this group of Diptera in Eastern Asia. including Korea, the material has been studied. Tachinine flies were collected during five expeditions to North Korea: in 1959, 1965, 1966, 1970 and in 1990. Detailed descriptions of these expeditions refer to Mroczkowski (1972) and in Bańkowska \& Sterzyńska (1997).

The scanty material of Tachininae includes 90 specimens collected with a sweeping net or using Moericke's yellow traps placed on grass and in brushwood. The locations of the sampling areas in North Korea are presented in Fig. 1.

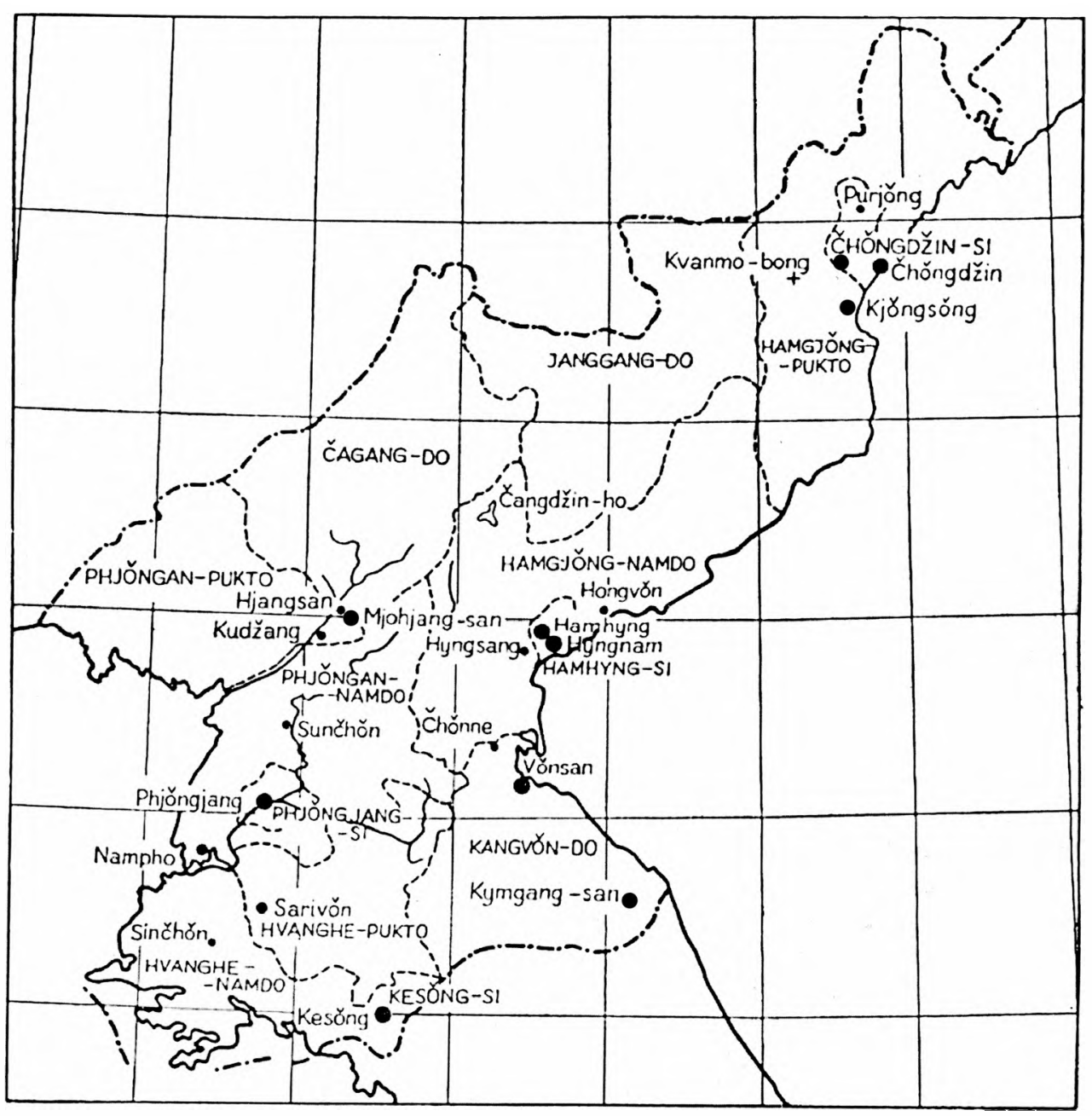

Fig.1. Location of sampling areas in North Korea in the years 1959-1990. 


\section{Subfamily Tachininae}

Tribe Ernestiini

Linnaemya Robineau-Desvoidy, 1830

Linnaemya (Linnaemya) Robineau-Desvoidy, 1830

\section{Linnaemya (Linnaemya) atriventris (Malloch, 1935)}

Palpina atriventris Malloch, 1935: 580. Type locality: Malaysia, Malay Peninsula, Pahang, Cameron Highlands, Rhododendron Hill, $5200 \mathrm{ft}$.

Linnaemyia montshadskyi Zimin, 1954: 272. Type locality: Russia, Primorskiy Kray, near Shkotovo, Kamenushka.

Distribution: Palaearctic: Japan (Hokkaidō, Honshū, Kyūshū), South Korea, Russia (S Far East), China (Inner Mongolia, Sichuan Prov., Shanxi Prov., Tibet); Oriental: Indonesia (Jawa), Malaysia (Pen. Malaysia), Thailand (O’Hara, Shima \& Zhang 2009).

\section{Linnaemya (Linnaemya) comta (Fallén, 1810)}

Tachina comta Fallén, 1810: 277. Type locality: Sweden

Material examined: North Korea, Chŏngdžin-si Prov., 10.09.1970, 1 male, leg. R. Bielawski and M. Mroczkowski.

Distribution: Palaearctic: Europe northwards to Shetland Islands and Middle Sweden, the European part of Russia, Ukraine, Transcaucasia, W and E Siberia, Russian Far East, Kuril Islands (Kunashir), Middle East, Afganistan, Mongolia, China (distributed almost all over the country, but has not been recorded from: the Provinces: Beijing Municipality, Chongqing Municipality, Guangdong, Guizhou, Hainan, Hong Kong S.A.R., Hunan and Macao S.A.R.); Oriental Region: Taiwan, India, Nepal; Afrotropical Region: Sudan. Nearctic Region: widespread (Shima 1986, Ziegler \& Shima 1996, Richter 2004 and O'Hara, Shima \& Zhang 2009). In Korea not mentioned up to the present.

\section{Linnaemya (Linnaemya) hirtipennis Shima, 1986}

Linnaemya (Linnaemya) hirtipennis Shima, 1986: 80. Type locality: Japan, Hokkaidō, Ashoro, Kiyokawa

Distribution: Palaearctic: Japan (Hokkaidō), South Korea (Lim \& Han 2008).

Linnaemya (Ophina) Robineau-Desvoidy, 1863

\section{Linnaemya (Ophina) microchaetopsis Shima, 1986}

Linnaemya microchaetopsis Shima, 1986: 35. Type locality: Japan, Kyūshū, Fukuoka City, Mt. Aburayama.

Material examined: North Korea, Chŏngdžin-si Prov., Kjǒngsǒng distr., Onpho-ri ad Chŏngdžin, 14.08.1959, 1 male; 18.08.1959, 1 male, 2 females, leg. B. Pisarski and J. Prószyński. Phjŏngan-pukto Prov., Mjohjang-san Mts., near Mjohjang-san hotel, 912.06.1990, 1 female, leg. E. Chudzicka, E. Kierych and R. Pisarska. Kangvŏn-do Prov., Ungčin, 25 km S of Vŏnsan, 18.09.1970, 1 male, leg. R. Bielawski and M. Mroczkowski.

Distribution: Palaearctic: Central Asia, Russia (S Far East), Japan (Hokkaidō, Honshū, Shikoku, Kyūshū), South Korea, China (distributed almost all over the country, but has not been recorded from two Provinces: Henan and Hebei and Macao Special Administrative Region); Oriental Region: Taiwan (Ziegler \& Shima 1996, Hua 2006, O'Hara, Shima \& Zhang 2009). 


\section{Linnaemya (Ophina) picta (Meigen, 1824)}

Tachina picta Meigen, 1824: 261. Type locality: not given (Europe, from "Baumhauerische Sammlung") Linnaemya retroflexa Pandellé, 1895: 350. Type localities: France, Hautes-Pyrénées (Tarbes) and Landes (Dux)

Material examined: North Korea, Chŏngdžin-si Prov., Kjŏngsǒng distr., Onpho-ri ad Chŏngdžin, 18.08.1959, 1 male, leg. B. Pisarski and J. Prószyński. Hamgjŏng-pukto Prov., distr. Kjŏngsŏng, Onpho-ri, 8.09.1966, 2 females, leg. C. Dziadosz and H. Szelęgiewicz.

Distribution: Palaearctic: Europe, Transcaucasia, Siberia, Russian Far East (Amuria, Primorie, Kamchatka), S Kuril Islands (Shikotan), Japan (Hokkaido, Honshū, Shikoku, Kyūshū), China (distributed almost all over the country, but has not been recorded from Provinces: Hainan, Hebei, Henan, Hong Kong S.A.R. and Tianjin Municipality); Oriental Region: Taiwan, India, Nepal, Tailand (Shima 1986, Ziegler \& Shima 1996, Richter 2004 and O'Hara, Shima \& Zhang 2009). In Korea not mentioned up to the present.

\section{Linnaemya (Ophina) zachvatkini Zimin, 1954}

Linnaemyia zachvatkini Zimin, 1954: 276. Type locality: Russia, Primorskiy Kray, Okeanskaya

Material examined: North Korea, Hamgjŏng-pukto Prov., distr. Džuyr, Onpho-ri, 5.09.1970, 1 female, leg R. Bielawski and M. Mroczkowski.

Distribution: Palaearctic: W, S and E Europe, E Siberia, Russian Far East (Primorie), S Kuril Islands (Kunashir), Japan (Hokkaido, Honshū, Shikoku, Kyūshū), Mongolia, China (distributed almost all over the country, but has not been recorded from the Provinces:Anhui, Chongqing Municipality, Guangxi Zhuangzu A.R., Guizhou, Hainan, Hong Kong S.A.R., Hunan, Jiangsu, Jiangxi, Macao S.A.R., Ningxia Huizu A.R., Shandong, Shanghai Municipality, Shaanxi, Zhejiang) (Shima 1986, Ziegler \& Shima 1996, Richter 2004 and O'Hara, Shima \& Zhang 2009). In Korea not mentioned up to the present.

Eloceria Robineau-Desvoidy, 1863

\section{Eloceria delecta (Meigen, 1824)}

Tachina delecta Meigen, 1824: 349. Type locality: not given (probably Hamburg, Germany)

Material examined: North Korea, Phjŏngan-pukto Prov., Mjohjang-san Mts. at the foot of Hjangro Peak, 9-12.06.1990, 1 male, leg. E. Chudzicka, E. Kierych and R. Pisarska.

Distribution: Palaearctic: Europe: northwards to England and Middle Sweden, European part of Russia, Ukraine, Transcaucasia, Middle East, Israel, Central Asia, Mongolia, S Siberia (Altai, Tuva, Chita), Amuria, Primorie) (Richter 2004). In Korea not mentioned up to the present.

Tribe Germariochaetini

Germariochaeta Villeneuve, 1937

Germariochaeta clavata Villeneuve, 1937 (Figs 2-4)

Germariochaeta clavata Villeneuve, 1937: 7. Type locality: China, Jiangsu Prov., Suzhou [as "Soochow"]

Material examined: North Korea, Phjǒngjang-si Prov., Phjǒngjang, 11.09.1959, 1 male, leg. B. Pisarski. 
Distribution: Palaearctic: Russia (South Part of Primorie) (Richter 2004); China (Fujian Prov.. Hebei Prov.. Heilongjiang Prov. and Jiangsu Prov.) (O’Hara. Shima \& Zhang 2009). In Korea not mentioned up to the present.

2

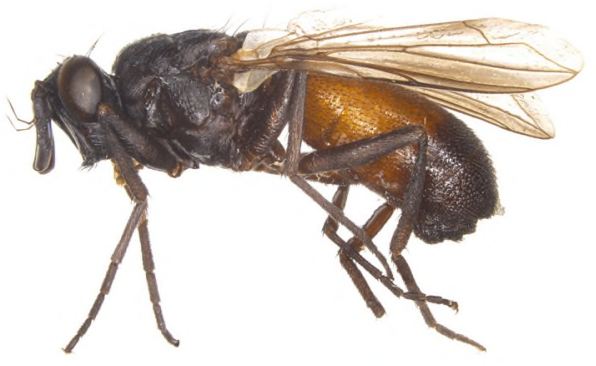

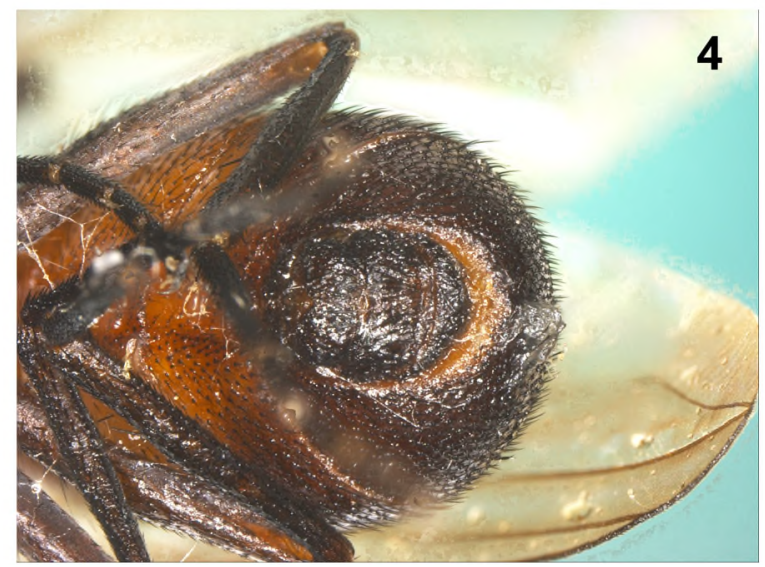

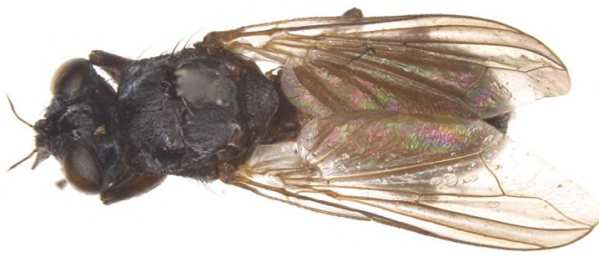

Figs 2-4. Germariochaeta clavata Villeneuve, male (photo P. Ślipiński); 2 - habitus in lateral view, 3 - habitus in dorsal view, 4 - abdomen in back view.

Tribe Leskiini

Atylostoma Brauer et Bergenstamm. 1889

\section{Atylostoma tricolor (Mik, 1884)}

Leskia tricolor Mik, 1884: 257. Type locality: Austria, Hamburg on Danube

Material examined: North Korea, Chŏngdžin-si Prov., Kjŏngsŏng distr., Onpho-ri ad Chŏngdžin, 18.08.1959, 1 female, leg. B. Pisarski and J. Prószyński.

Distribution: Palaearctic: Europe: France, Belgium. Switzerland. Austria, Slovenia, Slovakia, Poland. Belorussia. N Russia. Transcaucasia, Russian Far East: Primorie, Sakhalin. Kuril Islands (Kunashir), Japan (Hokkaidō) (Herting \& Dely-Draskovitz 1993. Draber-Mońko 1995. Ziegler \& Shima 1996. Bystrowski 1997, Richter 2004). In Korea not mentioned up to the present. 
Nigara Richter. 1999

\section{Nigara gracilis Richter,1999 (Fig. 5)}

Nigara gracilis Richter,1999: 726. Type locality: Russian Far East, Primorie, Chasanskii region, Lebedinoye.

Material examined: North Korea. Kangvön-do Prov.. Kumgang-san Mts.. Onjong-ri near Kumgang-san hotel. 23-25.06.1990, 1 male. leg. E. Chudzicka. E. Kierych and R. Pisarska.

Distribution: Russian Far East (South part of Primorie) (Richter 2004). In Korea not mentioned up to the present.

Note the difference: "eyes bare" in the description of the genus Nigara Richter, 1999: 724, but "eyes densely covered with long hairs" in Richter 2004: 342. The specimen from North Korea has eyes bare.

\section{5}

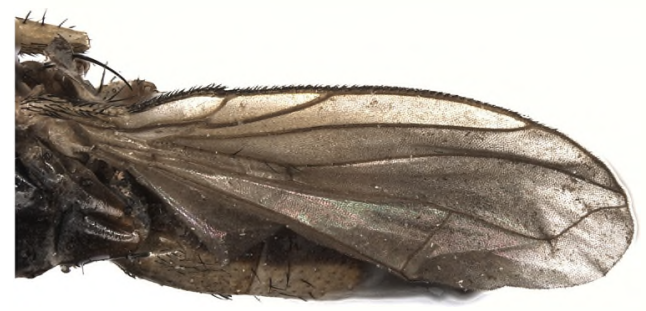

Fig. 5. Nigara gracilis Richter, male (photo P. Ślipiński); 5 - wing in view from above.

Tribe Macquartiini

Pseudebenia Shima. Han et Tachi. 2010

Pseudebenia epilachnae Shima et Han, 2010

Pseudebenia epilachnae Shima et Han, in Shima, Han \& Tachi, 2010: 54. Type locality: South Korea, Mt. Yongmunsan, Yangpyeong-gun, Gyeonggi-do.

Distribution: South Korea (Shima. Han \& Tachi, 2010).

Tribe Minthoini

Mintho Robineau-Desvoidy, 1830

\section{Mintho rufiventris (Fallén, 1817)}

Musca rufiventris Fallén, 1817: 239. Type locality: Sweden (Kalmar, Smäland).

Material examined: North Korea. Kangvốn-do Prov., Kumgang-san Mts., Onjong-ri near Kumgang-san hotel, 23-25.06.1990, 1 female, leg. E. Chudzicka, E. Kierych and R. Pisarska.

Distribution: Palaearctic: Europe northwards to S England and Middle Sweden, the European part of Russia. Ukraine. Transcaucasia. Israel. Middle East. Central Asia. Mongolia. S Siberia (Altai. Tuva, Chita), S Primorie (Ziegler \& Shima 1996, Richter 2004). In Korea not mentioned up to the present. 
Dolichopodomintho Townsend. 1927

\section{Dolichopodomintho takanoi Mesnil, 1973 (Fig. 6)}

Dolichopodomintho takanoi Mesnil, 1973: 1161.Type locality: Utatsuyama, Kanazawa, Honshū, Japan.

Material examined: North Korea. S Pyongan Prov., Pyongyang-City. Taesong Mts. near Michon Lake. 31.08.1987. 1 female, leg. E. Kierych.

Distribution: Palaearctic: Japan (Honshū. Shikoku. Kyūshū) (Mesnil 1973. Richter 2004). In Korea not mentioned up to the present.

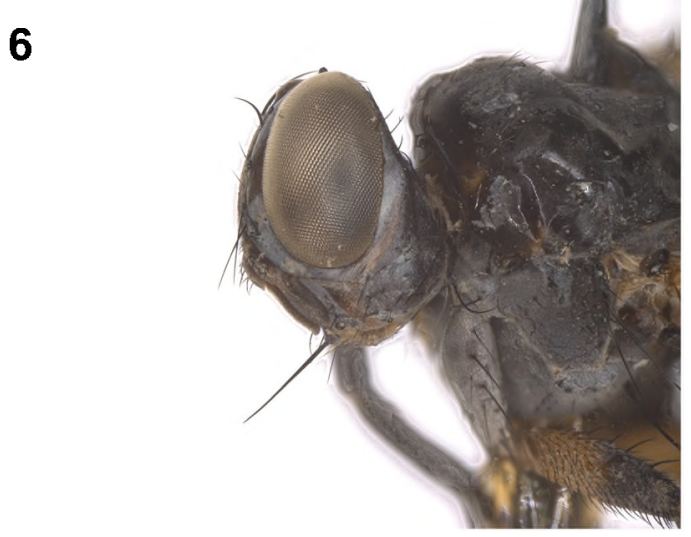

Fig. 6. Dolichopodomintho takanoi Mesnil, female (photo P. Slipiński); 6 - head with antenna in profile.

Remarks: In the first version of this paper I have given the information that among the specimens examined I found female belonging to the Dolichopodomintho dolichopiformis Townsend. 1927 (after the Key to the insects of Russian Far East. Vol. VI. Diptera and Siphonaptera. Pt 3. Vladivostok. 2004. 124. Fam. Tachinidae. Richter: 344-345). Professor $\mathrm{H}$. Shima, one of reviewers, in his comments said: "So far as looked at Fig. 6. this is more likely $D$. takanoi. It shows reddish basal $1 / 2$ of fore femora which is characteristic to takanoi".

During our subsequent correspondence we compared the morphological characters of my North Korean specimen with the Japanese D. takanoi Mesnil from Prof. H. Shima's collection.

Professor H. Shima has examined specimens of Japanese D. takanoi Mesnil and compared them with Dolichopodomintho dolichopiformis Townsend and stated the following: "Females of $D$. takanoi are similar to D. dolichopiformis and sometimes very similar. They appear to have some variations in colour and sometimes it is difficult to identify them".

Thanks to the comments of Prof. H. Shima and after my repeated study of the specimen from North Korea. I decided that it is D. takanoi Mesnil.

My specimen from North Korea has the following characteristics: taster and basicosta yellow; halteres at base (scabellum) and stem (pedicel) yellow, knob (capitulum) yellowbrown; costal spine as long as second part of costa; wing light brown with fuscus spot in apical part, lower calypter transparent but light brown; f1 at base red, $\mathrm{f} 2$ and $\mathrm{f} 3$ completely red; ground colour of abdomen black with whitish-bluish microtrichosity, which becomes more conspicuous in the posterior view. 
Tribe Nemoraeini

Hystriomvia Portschinsky, 1881

\section{Hystriomyia nigrosetosa Zimin, 1931 (Figs 7-8)}

Hystriomyia nigrosetosa Zimin, 1931: 34. Type locality: Mongolia, Ōmnōgovǐ Aimag [as "Zentral-Gobi"], near Gurvan Sayan Mts.["as Dundu-Sajchangebirge"], Ulan-Bulak [as "Ulan-Bulyk"].

Material examined: North Korea. Chŏngdžin-si Prov., Kjŏngsŏng distr., Onpho-ri ad Chŏngdžin. 18.08.1959. 1 male. leg. B. Pisarski and J. Prószyński.

Distribution: Palaearctic: Mongolia, Russia (W Siberia, S Far East). China (Inner Mongolia. Hebei Prov.. Sichuan Prov., Shaanxi Prov.. Yunnan Prov.) (Richter 2004, O'Hara. Shima \& Zhang 2009). In Korea not mentioned up to the present.

Note: The Korean specimen has two proclinate, strong orbital setae. 3 katepisternal setae, $4+4$ dorsocentral setae and 4+3 acrostichal setae. Cerci. surstyli and V sternite are very similar to those in the drawings 1 and 2 on page 474 in (Chao 1974).
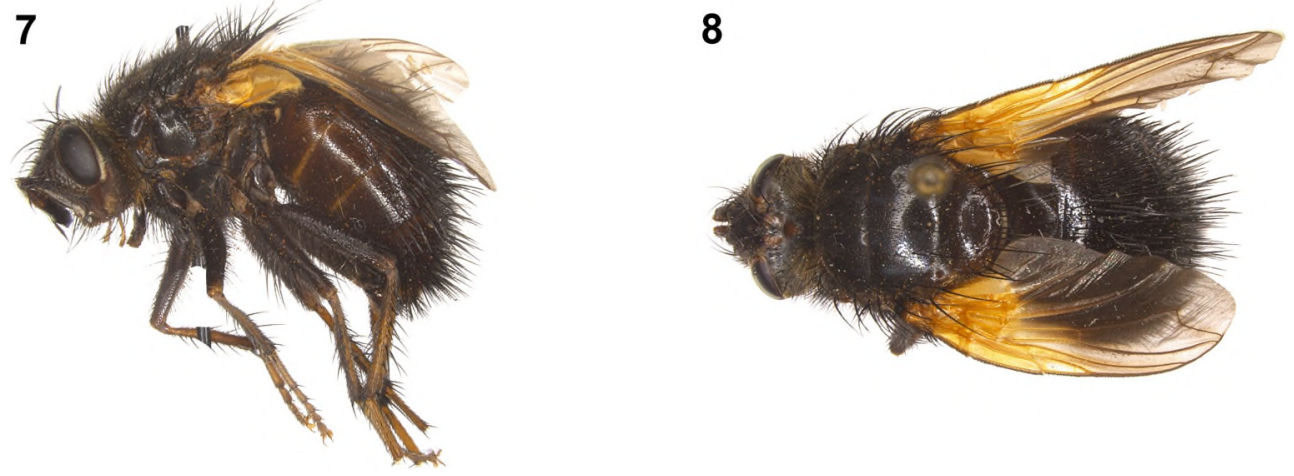

Figs 7-8. Hystriomyia nigrosetosa Zimin, male (photo P. Ślipiński); 7 - habitus in lateral view, 8 - habitus in dorsal view.

Tribe Palpostomatini

Eutrixopsis Townsend, 1919

\section{Eutrixopsis javana Townsend, 1919}

Eutrixopsis javana Townsend, 1919: 166. Type locality: Indonesia, Java, Ratoe, Palaboean.

Distribution: Palaearctic: Japan (Hokkaidō. Honshū. Shikoku. Kyūshū), Korea, Oriental Region: Japan (Ryuku Is.). China (Guangxi Zhuangzu Autonomous Region). Indonesia (Jawa). Malaysia (E Malaysia) (Richter 2004. O’Hara, Shima \& Zhang 2009).

Hamaxia Walker, 1860

\section{Hamaxia incongrua Walker, 1860}

Hamaxia incongnua Walker 1860: 153. Type locality: Indonesia, Maluku Islands, Ambon Islands [as "Amboyna"].

Distribution: Palaearctic: Russia (Siberia, Primorie), S-E Asia, Korea, Japan (Hokkaidō, Honshū. Kyūshū), China (Shandong Prov.); Oriental Region: China (Fujian Prov., Taiwan), 
Indonesia (Jawa, Sumatra), Malaysia (Malaya Peninsula) (Richter 2004, Hua 2006, O’Hara, Shima \& Zhang 2009).

Tribe Siphonini

Actia Robineau-Desvoidy, 1830

\section{Actia ampla Tachi et Shima, 1998}

Actia ampla Tachi et Shima, 1998: 449. Type locality: Japan, Kitamata-rindô, Nara.

Distribution: Palaearctic: Japan (Hokkaidō, Honshū), South Korea (Tachi \& Shima, 1998).

\section{Actia crassicornis (Meigen, 1824)}

Tachina crassicornis Meigen:351. Type locality: not given (probably Germany, Stolberg).

Material examined: North Korea, Kangvǒn-do Prov., Kūmgang-san Mts., Onjong-ri near Kūmgang-san hotel, 23-25.06.1990, 4 males, 1 female, leg. E. Chudzicka, E. Kierych and R. Pisarska. Phjŏngan-pukto Prov., Mjohjang-san Mts. at the foot of Hjangro Peak, 912.06.1990, 2 males, leg. E. Chudzicka, E. Kierych and R. Pisarska. Chŏngdžin-si Prov., Kjŏngsŏng distr., Onpho-ri ad Chŏngdžin, 14-16.08.1959, 1 male, leg. B. Pisarski and J. Prószyński.

Distribution: Palaearctic: Europe (all), Transcaucasia, Kazahstan, Mongolia, Russia (W Russia, E Siberia, S Far East), Japan (Hokkaidō, Honshū), China (Beijing Municipality, Hainan Prov., Jilin Prov. and Shanxi Prov.) (Ziegler \& Shima 1996, Tachi \& Shima 1998, Richter 1993, 2004, O’Hara, Shima \& Zhang 2009). In Korea not mentioned up to the present.

Ceromya Robineau-Desvoidy, 1830

\section{Ceromya bicolor (Meigen, 1824)}

Tachina bicolor Meigen, 1824: 354. Type locality: not given (probably Germany, Stolberg).

Material examined: North Korea, Phjŏngan-pukto Prov., Mjohjang-san Mts. at the foot of Hjangro Peak, 9-12.06.1990, 2 males, leg. E. Chudzicka, E. Kierych and R. Pisarska.

Distribution: Palaearctic: Europe northwards to Sweden, European part of Russia, E Siberia, Transcaucasia, China (Inner Mongolia) (Richter 2004, O'Hara, Shima \& Zhang 2009). In Korea not mentioned up to the present.

\section{Ceromya helvola Tachi et Shima, 2000}

Ceromya helvola Tachi et Shima, 2000: 140. Type locality: Japan, Hokkaidō, Yubaridate, Sorachi.

Distribution: Palaearctic: Russia (S Far East), Korea, Japan (Hokkaidō, Honshū, Kyūshū) (Tachi \& Shima 2000).

\section{Ceromya silacea (Meigen, 1824)}

Tachina silacea Meigen, 1824: 355. Type locality: not given (Europe, from "Baumhauerische Sammlung").

Material examined: North Korea, Kangvŏn-do Prov., Kūmgan-san Mts., 22.06.1990, 1 male, 2 females, leg. E. Kierych. 
Distribution: Palaearctic: Europe northwards to S England, Sweden, N European part of Russia, Transcaucasia, S Siberia, S Far East, Kuril Islands (Kunashir, Shikotan), Korea, Japan (Hokkaidō, Honshū, Shikoku, Kyūshū), China (Anhui Prov., Beijing Municipality, Fujian Prov., Henan Prov., Heilogjiang Prov., Hubei Prov., Liaoning Prov., Shanghai Municipality, Yunnan Prov.); Oriental Region: Japan (Ryukyu Is.), Taiwan (Tachi \& Shima 2000, Richter 2004, O’Hara, Shima \& Zhang 2009).

Proceromyia Mesnil, 1957

Nipponoceromyia Mesnil et Shima, 1978

\section{Proceromyia pubioculata (Mesnil et Shima, 1978)}

Nipponoceromyia pubioculata Mesnil et Shima, 1978: 324. Type locality: Japan (Honshū), Kawaragoya, Shigakogen, Nagano.

Distribution: Palaearctic: Japan (Honshū, Kyūshū), South Korea (Tachi \& Shima 2006).

Peribaea Robineau-Desvoidy, 1863

\section{Peribaea abbreviata Tachi et Shima, 2002}

Peribaea abbreviata Tachi et Shima, 2002: 121. Type locality: Japan, Honshū, Gifu Pref., Hodaka, $1100 \mathrm{~m}$.

Distribution: Palaearctic: Japan (Hokkaidō, Honshū, Shikoku, Kyūshū), South Korea, China (Guangdong Prov., Shaanxi Prov.) (Tachi \& Shima 2002, O’Hara, Shima \& Zhang 2009).

\section{Peribaea caesiata Tachi et Shima, 2002}

Peribaea caesiata Tachi et Shima, 2002: 133. Type locality: Japan, Hyôgo Pref., Onsen Town, Ueyama-kôgen.

Distribution: Palaearctic: Japan (Honshū, Kyūshū), South Korea (Tachi \& Shima 2002, Richter 2004).

\section{Peribaea setinervis (Thomson, 1869)}

Thryptocera setinervis Thomson, 1869: 519. Type locality: China.

Thryptocera fissicornis Strobl, 1910: 139. Type localities: Austria, Admont and Innsbruck.

Material examined: North Korea, Phjŏngan-pukto Prov., Mjohjang-san Mts. at the foot of Hjangro Peak, 9-12.06.1990, 1 male, leg. E. Chudzicka, E. Kierych and R. Pisarska.

Distribution: Palaearctic: Europe northwards to England and Scotland, S Scandinavia; Ukraine, W Siberia, S Siberia and E Siberia, Russian Far East (Amuria, Primorie), Japan (Hokkaidō, Honshū, Kyūshū), China (Guangdong Prov., Hong Kong S.A.R., Zhejiang Prov.); Oriental Region: Myanmar (Richter 2004, O’Hara, Shima \& Zhang 2009). In Korea not mentioned up to the present.

\section{Peribaea tibialis (Robineau-Desvoidy, 1851)}

Herbstia tibialis Robineau-Desvoidy, 1851: 185. Type locality: not given (France, probably near Paris).

Material examined: North Korea, Phjŏngan-pukto Prov., Mjohjang Mts., 5.08.1959, 1 female, leg. B. Pisarski and J. Prószyński.

Distribution: Palaearctic: widely distributed in S and C Europe (from Portugal, Spain, N Italy, Greece, France, Switzerland, German, Poland, Ukraine to Russian Karelia), Morocco, 
Asia: Israel, Turkey, Transcaucasia, Uzbekistan, $\mathrm{M}$ and E Mongolia, S Russian Far East, S Korea, Japan (Hokkaidō, Honshū, Kyūshū), China (Beijing Municipality, Fujian Prov., Guangdong Prov., Guizhou Prov., Hainan Prov., Sichuan Prov., Shaanxi Prov., Shanxi Prov., Yunnan Prov., Zhejiang Prov., Hong Kong S.A.R.); Oriental Region: Taiwan, Japan (Ryukyu Is.), Myanmar. Afrotropics: ? Democratic Republic of the Congo, Kenya, ? South Africa (Andersen 1996, Ziegler \& Shima 1996, Richter 2004, O’Hara, Shima \& Zhang 2009).

\section{Entomophaga Lioy, 1864}

\section{Entomophaga nigrohalterata (Villeneuve, 1921)}

Actia nigrohalterata Villeneuve, 1921: 45. Type locality: Denmark, East Jutland.

Actia articulate Stein, 1924: 131. Type locality: Denmark (Copenhagen) and Germany (Löbauer Berg, Oberlausitz).

Material examined: North Korea, N Hamgyong Prov., Chongjin-City, Kyowon-ri, 16.06.1990, 1 female, leg. E. Chudzicka, E. Kierych and R. Pisarska.

Distribution: Palaearctic: Europe northwards to S Sweden, Russia (South of Primorie), Japan (Honshū) (Richter 2004, Tachi \& Shima 2006). In Korea not mentioned up to the present.

Siphona Meigen, 1803

\section{Siphona confusa Mesnil, 1961}

Siphona confusa Mesnil, 1961: 201. Type locality: Sweden, Lake Vättern, Gränna.

Material examined: North Korea, Kangvŏn-do Prov., Vǒnsan, public park and neighbouring hills, 29.08.1987, 2 females, leg. E. Kierych; Kūmgang-san Mts., Okryn Valley, 22.06.1990, 1 male, 1 female, leg. E. Chudzicka, E. Kierych and R. Pisarska; Kümgang-san Mts., Onjong-ri near Kūmgang-san hotel, 23-25.06.1990, 2 females, leg. E. Chudzicka, E. Kierych and R. Pisarska; Kūmgang-san Mts., 22.06.1990, 2 males, leg. E. Kierych; N Kwanghae Prov., Kaesong near hotel, 28.06.1990, 5 females, leg. E. Chudzicka, E. Kierych and R. Pisarska; Kaesong Park near Koryo Museum, 29.06.1990, 3 females, leg. E. Chudzicka, E. Kierych and R. Pisarska; Kaesong Park, 12.08.1987, 1 male, leg. E. Kierych; N Hamgyong Prov. Kyongsong country, Sangonpo-ri, 17.06.1990, 2 females, leg. E. Chudzicka, E. Kierych and R. Pisarska; S Pyongan Prov., Pyongyang City, Taesong Mts., near Michon Lake, 31.06.1987, 1 female, leg. E. Kierych.

Distribution: Palaearctic: Europe northwards to Scandinavia, Israel, Middle East, North Africa, Mongolia, Russia (W Russia, E Siberia), China (Fujian Prov., Gansu Prov., Heilongjiang Prov., Jilin Prov., Inner Mongolia, Qinghai Prov., Sichuan Prov., Xinjiang Uygur Autonomous Region, Tibet, Yunnan Prov.) (Richter 2004, O'Hara, Shima \& Zhang 2009). In Korea not mentioned up to the present.

Tribe Glaurocarini

Glaurocara Thomson, 1869

\section{Glaurocara lucidula Richter, 1988}

Glaurocara lucidula Richter, 1988: 204. Type locality: South Korea, Cheju-do (Quelpart Is.).

Distribution: South Korea, Cheju-do Island (Richter 1988, 2004). 
Tribe Tachinini

Mikia Kowarz., 1885

Anaeudora Townsend, 1933

T'amanukia Baranov. 1935

\section{Mikia japanica (Baranov, 1935) (Figs 9-12)}

Tamanukia japanica Baranov, 1935: 551. Type locality: Japan, Hokkaidō, Obihiro.

Material examined: North Korea, Chagang-do Prov., Mjohjang-san Mts., 5.08.1959. 1 female, leg. B. Pisarski and J. Prószyński. [det. L. S. Zimin as Anaeudora japanica Baranov].

9

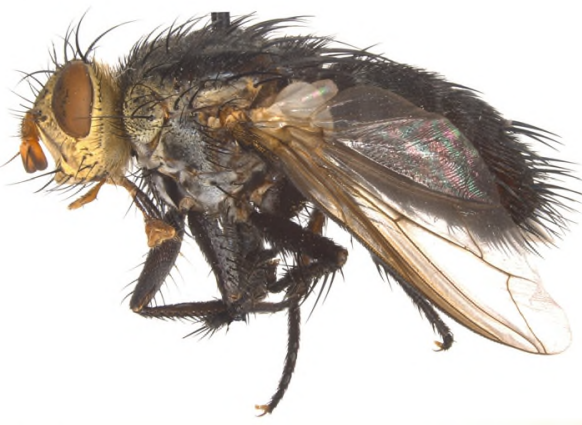

11

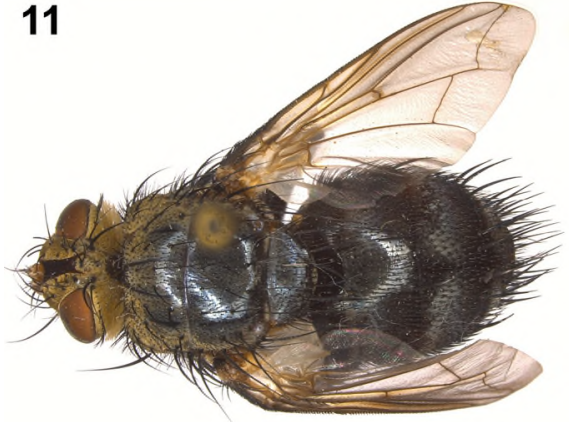

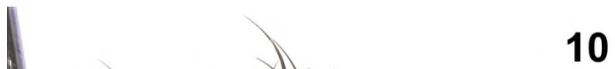
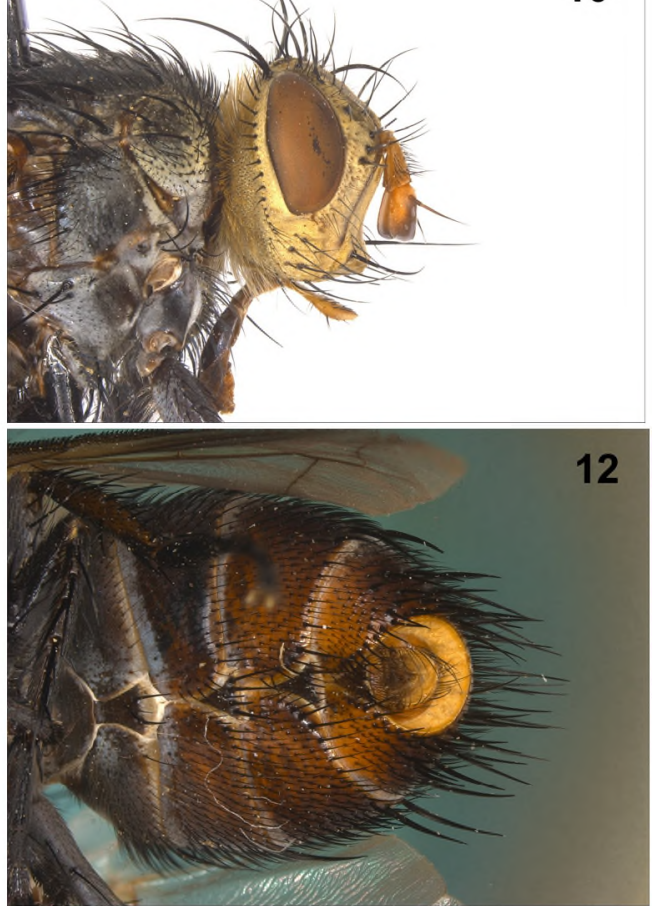

Figs 9-12. Mikia japanica (Baranov), female (photo P. Ślipiński); 9- habitus in lateral view, 10 - head with forder part of thorax, 11 - habitus in dorsal view, 12 -abdomen in ventral view.

Distribution: Palaearctic: Russian Far East (Primorie). Sakhalin. S Kuril Islands (Kunashir), Japan (Hokkaidō, Honshū. Kyūshū) (Richter 2004). China (Anhui Prov.. Fujian Prov.. Guangxi Zhuangzi A. R.. Hebei Prov.. Jilin Prov., Liaoning Prov.. Sichuan Prov.. Shaanxi Prov.. Yunnan Prov.); Oriental Region: Taiwan (Richter 2004. O'Hara. Shima \& Zhang 2009). In Korea not mentioned up to the present. 
Peleteria Robineau-Desvoidy, 1830

Cuphocera Macquart, 1845

Spyricera Lioy, 1864

\section{Peleteria iavana (Wiedemann, 1819)}

Tachina iavana Wiedemann, 1819: 24. Type locality: Indonesia, Java (Jakarta [as "Batavia"].

Musca varia Fabricius, 1794: 327 (junior primary homonym of Musca varia Gmelin, 1790). Type locality: "India orientali" [East Indies].

Distribution: Palaearctic: W, S and E Europe, W Russia, Kazakhstan, W and E Siberia, Russian Far East, Transcaucasia, North Africa (Tunisia), Japan (Hokkaidō, Honshū, Shikoku, Kyūshū), Korea, China (distributed almost all over the country, but has not been recorded from two provinces: Qinghai, Xinjiang Uygur A. R. and Macao S.A.R.); Oriental: Taiwan, India, Indonesia (Jawa, Sulawesi, Sumatra), Malaysia (Pen. Malaysia, E. Malaysia), Myanmar, Nepal, Philippines, Sri Lanka, Thailand; Australasian: Australia, Indonesia (Maluku Is.), Melanesia, Papua New Guinea; Afrotropical: northeastern to southern Africa, including Madagascar (Han \& Kim 1983, Richter 2004, O’Hara, Shima \& Zhang 2009).

\section{Peleteria pallida Zimin, 1935 (Figs 13-17)}

Peletieria (Peletieria) pallida Zimin, 1935: 612. Type locality: Russian Far East (Primorie).

Material examined: North Korea, Phjŏngan-pukto Prov., Mjohjang-san Mts., near Mjohjang-san hotel, 9-12.06.1990, 1 female, 11.06.1990, 1 female, leg. E. Chudzicka, E. Kierych and R. Pisarska; Mjohjang-san Mts., near Kumgang Cave, 10.06.1990, 1 female, leg. E. Chudzicka, E. Kierych and R. Pisarska.

Distribution: Palaearctic: Russian Far East (Khabarovsk, Primorie) (Richter 2004). In Korea not mentioned up to the present.

\section{Peleteria propinqua (Zimin, 1961)}

Hemipeletieria propinqua Zimin, 1961: 250. Type locality: Russian Far East (Primorie, Tasino Pass, Suchan).

Distribution: Palaearctic: Russian Far East, Korea, Japan (Hokkaidō); China (Liaoning Prov.) (Richter 2004, O’Hara, Shima \& Zhang 2009).

\section{Peleteria semiglabra (Zimin, 1961)}

Hemipeletieria semiglabra Zimin, 1961: 251. Type locality: Russian Far East, Khabarovsky Kray.

Peletieria pallida of Chao (1963: 220, as Hemipeletieria pallida) and Herting et Dely-Draskoits (1993: 278) not Zimin, 1935. Misidentification.

Distribution: Palaearctic: Russian Far East (Khabarovsky Kray, S Primorie), Korea, Japan (Honshū); China (Gansu Prov, NE China) (Richter 2004, O’Hara, Shima \& Zhang 2009).

\section{Peleteria sphyricera (Macquart, 1835)}

Echinomyia sphyricera Macquart, 1835: 78. Type locality: France, Bordeaux.

Material examined: North Korea, Phjŏngan-pukto Prov., Mjohjang-san Mts., 5.08.1959, 2 males, leg. B. Pisarski and J. Prószyński, [det. L. S. Zimin as Sphyricera sphyricera (Macquart)], Chǒngdžin-si Prov., Kjǒngsǒng distr., Onpho-ri ad Chǒngdžin, 18.08.1959, 2 females, leg. B. Pisarski and J. Prószyński, [det. L. S. Zimin as Sphyricera sphyricera (Macquart)]; Džuyr ad Chǒngdžin, 24.08.1959, 1 female, leg. B. Pisarski and J. Prószyński, 
[det. L. S. Zimin as Sphyricera sphyricera (Macquart)] and 1 male, leg. B. Pisarski and J. Prószyński.

Distribution: Palaearctic: Europe (W. S and E Europe). Transcaucasia. Russia (E Siberia. S Far East. Sakhalin). China (Beijing Municipality. Gansu Prov.. Hebei Prov.. Heilongjiang Prov., Liaoning Prov.. Inner Mongolia) (Richter 2004. O'Hara. Shima \& Zhang 2009). In Korea not mentioned up to the present.
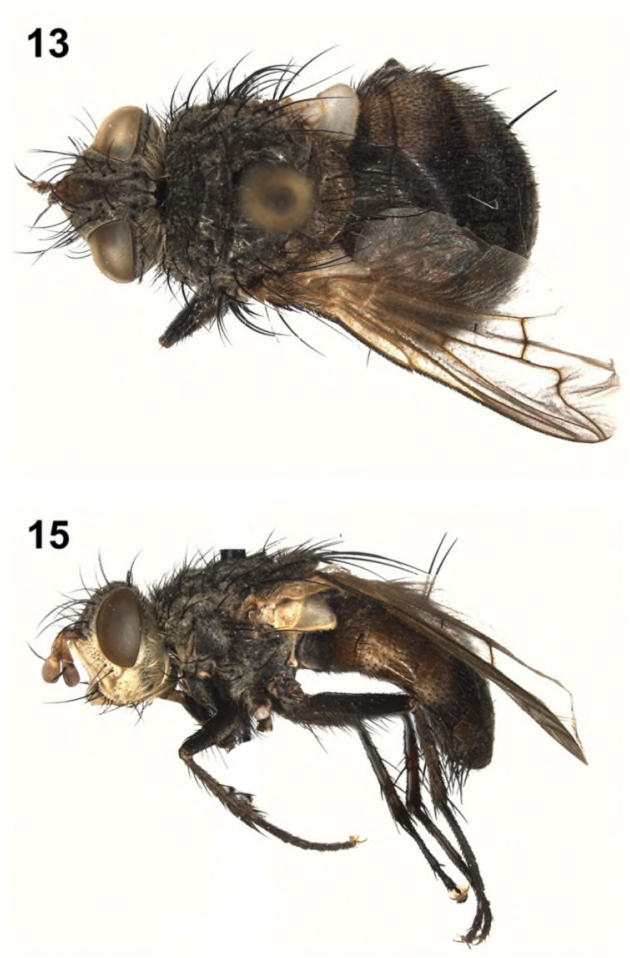

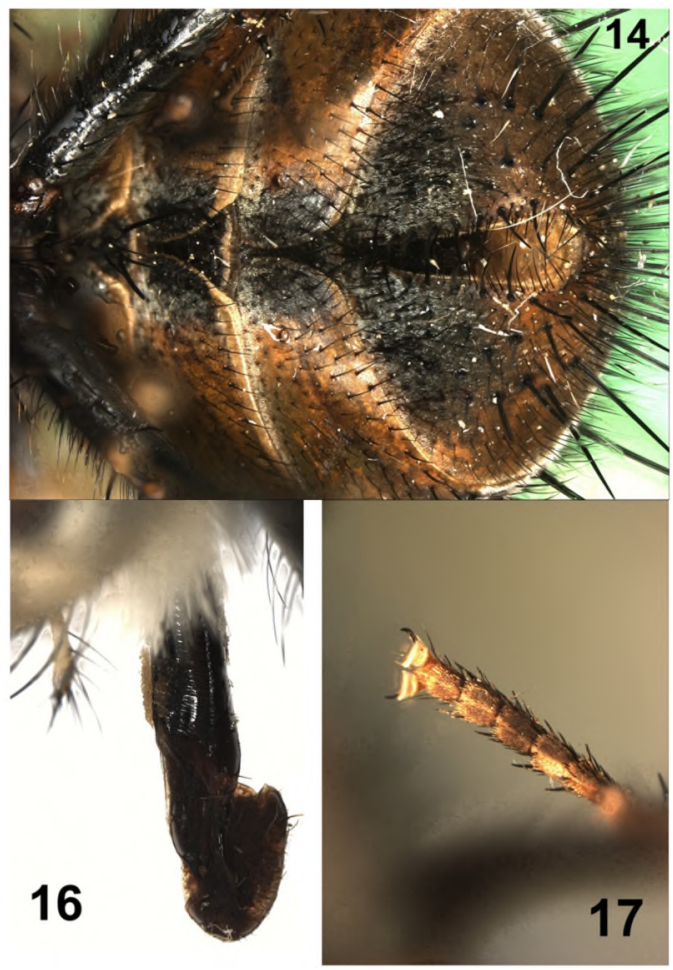

Figs 13-17. Peleteria pallida Zimin, female (photo P. Ślipiński); 13 - habitus in dorsal view, 14 - abdomen in ventral view, 15 - habitus in lateral view, 16 - palpus and proboscis in lateral view, 17 - fore tarsus.

Tachina Meigen. 1803

Tachina (Nowickia) Wachtl. 1894

Fabricia Robineau-Desvoidy, 1830

\section{Tachina (Nowickia) atripalpis (Robineau-Desvoidy, 1863)}

Fabricia atripalpis Robineau-Desvoidy, 1863: 627. Type locality: not given (France, probably near Paris).

Distribution: Palaearctic: Europe (W, S and E Europe). Transcaucasia. C Asia. Mongolia. Russia (W Russia. W and E Siberia. S Far East). China (Guangdong Prov.. Gansu Prov.. Heilongjang Prov.. Inner Mongolia. Qinghai Prov.. Sichuan Prov.. Shanxi Prov.. Xinjiang Uygur A.R.. Tibet. Zhejiang Prov.) (Richter 2004. O’Hara. Shima \& Zhang 2009). Korea (Hua 2006). 


\section{Tachina (Nowickia) marklini Zetterstedt, 1838}

Tachina marklini Zetterstedt, 1838: 634. Type locality: Lapland.

Distribution: Palaearctic: Europe (Central European Mts. and N Europe), Northern or mountain regions of Asia (Pamir and other mountains.), Mongolia. Siberia (W, C and E Siberia), Rusian Far East (Magadan. Amuria, Khabarovsk Territory, Ussuria. Sakhalin. Kuril Islands (Paramushir. Atlasova. Onekotan. Shiashkotan. Brat Tshirpojev). Kamchatka. Korea. China (Heilongjiang Prov.) (Richter 1986, 2004, Ziegler \& Shima 1996. O'Hara, Shima \& Zhang 2009).

\section{Tachina (Nowickia) rondanii (Giglio-Tos, 1890) (Figs 18-19)}

Echinomyia rondanii Giglio-Tos, 1790: 459. Type locality: Italy, Italian Alps, Piemont, Valli di Cuneo, Valdieri.

Material examined: North Korea, Chŏngdžin-si Prov., Kjǒngsǒng distr., Onpho-ri ad Chŏngdžin, 18.08.1959. 1 female, leg. B. Pisarski and J. Prószyński.

Distribution: Palaearctic: Europe (W and S Europe), Transcaucasia, Russia (the Central European part of Russia, W, S and E Siberia), C Asia, Kazakhstan. Mongolia, China (Inner Mongolia. Sichuan Prov., Shanxi Prov., Xinjiang Uygur A.R., Tibet, Yunnan Prov.) (Richter 2004. O'Hara. Shima \& Zhang 2009). In Korea not mentioned up to the present.

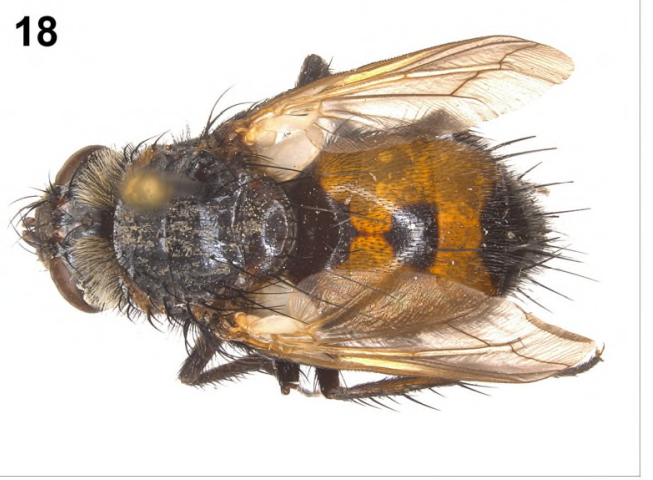

19

Figs 18-19. Tachina (Nowickia) rondanii (Giglio-Tos), female (photo P. Ślipiński); 18 - habitus In dorsal view, 19 head with thorax and forder part of abdomen in Lateran view.

Tachina (Tachina) Meigen. 1803

Echinomva Latreille. 1805

Servillia Robineau-Desvoidy, 1830

Tachina (Tachina) amurensis (Zimin, 1929)

Servillia amurensis Zimin, 1929: 218. Type locality: Russia, Amurskaya Oblast' [as "Amurgebiet"].

Distribution: Palaearctic: Russian Far East (Amur Prov., S Primorie). Sakhalin. S Kuril Islands (Kunashir), Korea, Japan (Hokkaidō. Honshū. Shikoku, Kyūshū); China (Inner Mongolia. Sichuan Prov.) (Zimin \& Kolomiets 1984. Ziegler \& Shima 1996. Richter 2004. O'Hara. Shima \& Zhang 2009). 


\section{Tachina (Tachina) ardens (Zimin, 1929) (Figs 20-21)}

Servillia ardens Zimin, 1929: 219. Type localities: Russian Far East, Primorskiy Kray (Sopka Kamenj, at village of Kamenj-Rybolov on Lake Chanka; Evgenievka Station) and China Gansu, Chojasan, $3000 \mathrm{ft}$.

Material examined: North Korea. Hamgjŏng-pukto Prov., distr. Džuyr, Onpho-ri, 6.09.1970, 2 females, leg. R. Bielawski and M. Mroczkowski, distr. Kjǒngsǒng. Onpho-ri, 10.09.1966, 2 females. leg. C. Dziadosz and H. Szelęgiewicz. Kangvǒn-do Prov., Vǒnsan. 20.09.1970, 1 females, leg. R. Bielawski and M. Mroczkowski.

Distribution: Palaearctic: Middle East. Russia: E Siberia. S Far East (Amur Prov.. $S$ Primorie); China (distributed almost all over the country, but has not been recorded from: the Provinces: Guangdong. Hainan. Hebei. Shanxi and Beijing Municipality. Hong Kong S.A.R.. Shanghai Municipality and Tianjin Municipality); Oriental Region: Taiwan. Myanmar (Richter 2004. O'Hara. Shima \& Zhang 2009). In Korea not mentioned up to the present.
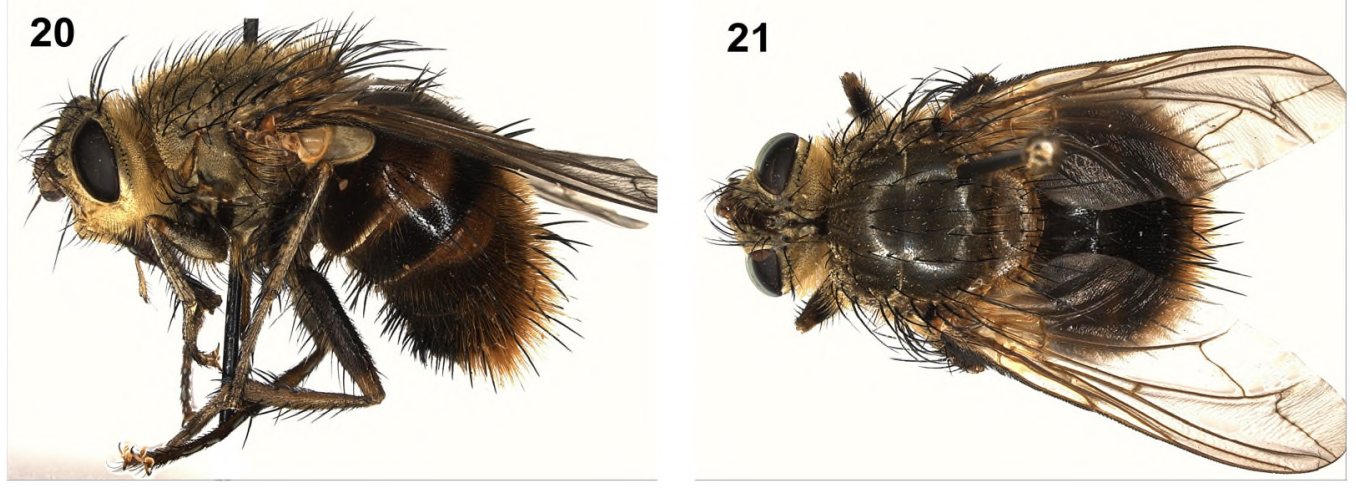

Figs 20-21. Tachina (Tachina) ardens (Zimin, female (photo P. Ślipiński); 20 - habitus in lateral view, 21 - habitus in dorsal view.

Tachina (Tachina) breviceps (Zimin, 1929)

Servillia breviceps Zimin, 1929: 214. Type locality: Russian Far East, Primorie, Yakovlevka [as "Jakovlevka"]. Servillia pallidohirta Zimin, 1929: 215. Type locality: Russian Far East, Primorie (Yakovlevka [as "Jakovlevka"]; Sutshan distr., Siza Station, Shkotovo distr., Mikhaylovka [as "Michailovka"]) and China ("West Manshurien: "Tshajano" (most probably Inner Mongolia, Chaoyangcun (O'Hara, Shima \& Zhang 2009).

Distribution: Palaearctic: Russia: E Siberia (Chita). Far East (Amuria, Khabarovsk Territory, Primorie, Sakhalin), Kuril Islands (Kunashir), Korea, Japan (Hokkaidō. Honshū); China (Anhui Prov., Beijing Municipality, Fujian Prov., Heilongjiang Prov., Jiangsu Prov., Jiangxi Prov., Inner Mongolia, Qinghai Prov., Sichuan Prov.. Shandong Prov., Shanghai Municipality, Shanxi Prov. Tibet. Yunnan Prov., Zhejiang Prov.); Oriental Region: Taiwan (Ziegler \& Shima 1996, Richter 2004, O’Hara. Shima \& Zhang 2009).

\section{Tachina (Tachina) fera (Linnaeus, 1761)}

Musca fera Linnaeus, 1761: 453. Type locality: Europe: Sweden.

Material examined: North Korea. Phjŏngan-namdo Prov., distr. Sunan. Sokam-Juvǒndži. 21.05.1965, 1 female. leg. R. Bielawski and M. Mroczkowski. Chǒngdžin-si Prov.. Kjŏngsǒng distr.. Onpho-ri ad Chŏngdžin. 18.08.1959. 1 female. leg. B. Pisarski and J. Prószyński. 
Distribution: Palaearctic: Europe (all), North Africa, Transcaucasia, C Asia, Middle East Mongolia, Russia (W Russia, W Siberia, E Siberia, S Far East), Japan (Hokkaidō, Honshū); China (Beijing Municipality, Hubei Prov., Jilin Prov., Inner Mongolia, Shanxi Prov., Tjanjin Municipality, Xinjiang Uygur A.R.,Tibet (Richter 2004, O'Hara, Shima \& Zhang 2009). In Korea not mentioned up to the present.

\section{Tachina (Tachina) iota Chao et Arnaud, 1993}

Servillia minuta Chao, 1962: 56. Type locality: Japan, Honshū, Nagano Pref., Mt. Iwasuge (junior secondary homonim of Tachina minuta Fallén, 1810).

Tachina iota Chao et Arnaud, 1993: 48 (nomen novum for minuta Chao, 1962).

Material examined: North Korea, Kesǒng-si Prov., Kesǒng-City, canyon at Pagyon Falls, 30.06.1990, 1 female, leg. E. Chudzicka, E. Kierych and R. Pisarska.

Distribution: Palaearctic: Japan (Hokkaidō, Honshū, Shikoku, Kyūshū), Russia: Sakhalin, South Kuril Islands (Iturup, Kunashir, Shikotan); China (Beijing Municipality, Henan Prov., Liaoning Prov., Inner Mongolia, Sichuan Prov.) (Richter 2004, O’Hara, Shima \& Zhang 2009). In Korea not mentioned up to the present.

\section{Tachina (Tachina) jakovlewii (Porschinsky, 1882)}

Echinomyia jakovlewii Portschinsky, 1882: 7. Type locality: Russian Far East, Amurskaya Oblast'.

Distribution: Palaearctic: Mongolia, Korea, Russia (Yakutskaya Resp., Chita, Amuria, S Primorie, Kuril Islands (Kunashir); China (Beijing Municipality, Gansu Prov., Hebei Prov., Heilongjiang Prov., Jilin Prov., Inner Mongolia, Shanxi Prov.) (Han \& Kim 1983, Richter 2004, O'Hara, Shima \& Zhang 2009).

\section{Tachina (Tachina) luteola (Coquillett, 1898)}

Servillia luteola Coquillett, 1898:329. Type locality: Japan, Honshu, Gifu.

Distribution: Palaearctic: Russia (S Far East), Korea, Japan (Hokkaidō, Honshū, Shikoku, Kyūshū); China (Heilongjiang Prov., Sichuan Prov., Zhejiang Prov.) (Han \& Kim 1983, Ziegler \& Shima 1996, Richter 2004, O’Hara, Shima \& Zhang 2009).

\section{Tachina (Tachina) macropuchia Chao, 1982}

Tachina macropuchia Chao in Chao \& Shi, 1982: 255. Type locality: China, Jilin Prov., Fusong.

Distribution: Palaearctic: Korea, China (Hebei Prov., Heilongjiang Prov., Jilin Prov., Liaoning Prov., Inner Mongolia, Shanxi Prov., Xinjiang Uygur A.R., Tibet) (O'Hara, Shima \& Zhang 2009).

\section{Tachina (Tachina) magnicornis (Zetterstedt, 1844)}

\footnotetext{
Echinomyia magnicomis Zetterstedt, 1844: 996. Type localities: Sweden, Östergötland and Gotland.

Tachina satanas Zimin, 1967: 476. Type locality: China, Nei Mongol, Khingan [presumably the Greater Khingan Range], Garnak.

Tachina vernalis of authors (e.g., Mesnil, 1966: 924, Chao, 1985: 125) not Robineau-Desvoidy, 1830. Misidentification.
}

Material examined: North Korea: Chagang-do Prov., Mjohjang-san Mts., 5.08.1959, 1 female, leg. B. Pisarski and J. Prószyński; Chŏngdžin-si Prov., Kjǒngsǒng distr., Onpho-ri ad Chǒngdžin, 14.08.1959, 1 male; 18.08.1959, 1 male, leg. B. Pisarski and J. Prószyński; Džuyr ad Chǒngdžin, 24.08.1959, 1 female, leg. B. Pisarski and J. Prószyński, det. L. S. Zimin. 
Distribution: Palaearctic: Europe (all), Transcaucasia, Israel, C Asia. Middle East Mongolia. Russia (W Russia. W and E Siberia. S Far East. Kuril Islands.(Kunashir). Korea. Japan (Hokkaidō); China (Beijing Municipality. Hebei Prov.. Heilongjiang Prov.. Jilin Prov.. Liaoning Prov.. Inner Mongolia. Ningxia Huizu A.R., Shanxi Prov.. Xinjiang Uygur A.R.) (Richter 2004, O’Hara. Shima \& Zhang 2009).

\section{Tachina (Tachina) majae (Zimin, 1935) (Figs 22-23)}

Servillia majae Zimin, 1935: 574. Type locality: Russian Far East ( Primorie), Suchan.

Material examined: North Korea. Phjŏngan-pukto Prov.. Mjohjang-san Mts.. near Kumgang Cave. 10.06.1990. 1 female. leg. E. Chudzicka. E. Kierych and R. Pisarska.

Distribution: Palaearctic: Russian Far East (South Primorie) (Zimin 1935, Zimin \& Kolomiets 1984, Richter 2004). In Korea not mentioned up to the present.
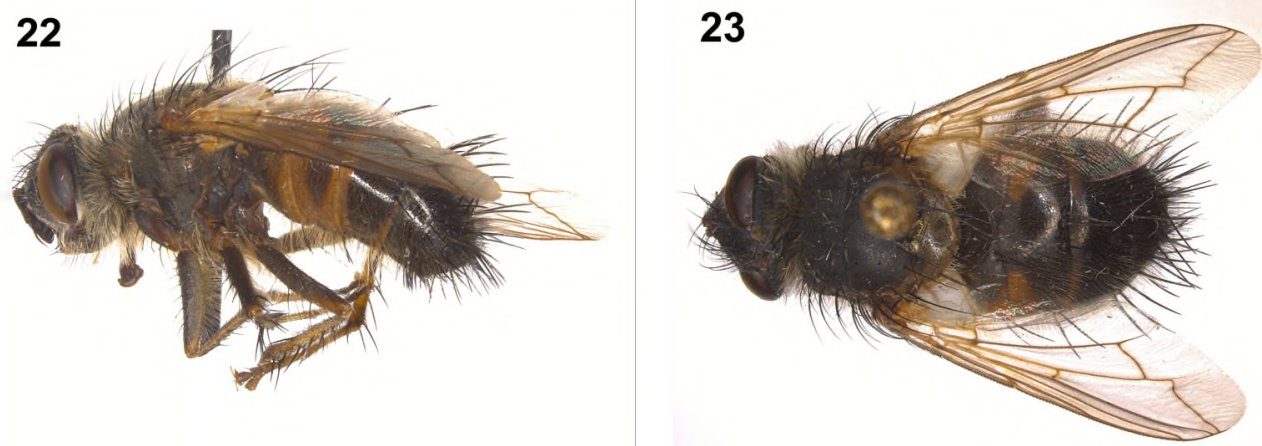

Figs 22-23. Tachina (Tachina) majae (Zimin), female (photo P. Ślipiński); 22 - habitus in lateral view, 25 - habitus in dorsal view.

\section{Tachina (Tachina) nupta (Rondani, 1859)}

Echinomya nupta Rondani, 1859: 55. Type locality: Italy

Echinomyia micado Kirby, 1884: 459. Type locality: Japan, Honshû, Kôbe.

Echinomyia trigonota Villeneuve, 1936: 3. Type locality: China, southern Gansu

Material examined: North Korea. Phjŏngan-pukto Prov., Mjohjang-san Mts., near Mjohjang-san hotel, 11.06.1990, 3 males, leg. E. Chudzicka. E. Kierych and R. Pisarska; Mjohjang-san Mts., near Mjohjang-san hotel, canyon. 11.06.1990, 2 males, leg. E. Chudzicka. E. Kierych and R. Pisarska; Mjohjang-san Mts..Tephun ad Kujang-dong. 4.09.1959, 1 female. leg. B. Pisarski and J. Prószyński; Chŏngdžin-si Prov., Kjŏngsŏng distr., Onpho-ri ad Chŏngdžin, 14.08.1959, 1 male, 18.08.1959. 2 females, leg. B. Pisarski and J. Prószyński; Hamgjŏng-pukto Prov.. Džuyr distr., Onpho-ri, 5.09.1970, 1 male. 2 females, leg. R. Bielawski and M. Mroczkowski; Hamhyng Prov.. Mačŏn. seaside locality, 20 km NE of Hamhyng. 26.09.1970, 1 female, leg R. Bielawski and M. Mroczkowski.

Distribution: Palaearctic: Europe (S Europe. E Europe), Transcaucasia. C Asia. Mongolia. Russia (W and E. Siberia) Russian Far East (Khabarovsk. Primorie. S. Kuril Islands (Kunashir). Korea. Japan (Hokkaidō. Honshū (Tsushima), Shikoku. Kyūshū); China (Beijing Municipality, Guangdong Prov.. Gansu Prov.. Zhuangzu A.R.. Hebei Prov.. Heilongjiang Prov.. Hubei Prov.. Jilin Prov.. Liaoning Prov.. Inner Mongolia. Ningxia Huizu A.R., Quighai Prov.. Sichuan Prov.. Shaanxi Prov.. Shanxi Prov.. Tianjin Municipality. Xinjiang Uygur A.R., Tibet. Yunnan Prov.. Zhejiang Prov.) (Han \& Kim 1983. Zimin \& Kolomiets 1984. Richter 2004. O'Hara. Shima \& Zhang 2009). 


\section{Tachina (Tachina) politula (Coquillett, 1898)}

Servillia politula Coquillett, 1898: 21. Type locality: Japan, Honshū, Gansu.

Servillia suzuki Matsumura, 1916: 391. Type locality: Japan, Honshâ, Kyoto. 1993).

Distribution: Palaearctic: Japan. Korea (Han \& Kim 1983. Herting \& Dely-Draskovits

\section{Tachina (Tachina)? stackelbergiana Herting et Dely-Draskovits, 1993}

Tachina stackelbergiana Herting et Dely-Draskovits, 1993: 267. Replacement new name of Tachina stackelbergi Zimin, 1935: 562, a junior secondary homonym of Servillia stackelbergi Zimin, 1929: 216. Type locality: Russian Far East.

Tachina zimini Kolomiets, 1975: 26 (new name for Tachina stackelbergi Zimin, 1935). A junior secondary homonym of Servillia zimini Chao, 1962

Material examined: North Korea, Phjŏngan-pukto Prov.. Mjohjang-san Mts.. Tephun ad Kujang-dong, 4.09.1959, 1 female, leg. B Pisarski, [det. L.S. Zimin as Tachina sp.].

\section{4}

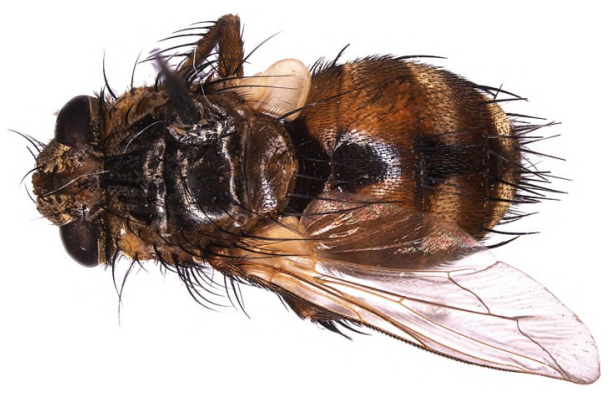

Fig. 24. Tachina (Tachina)? stackelbergiana Herting et Dely-Draskovits, female (photo P. Ślipiński); 24 - habitus in dorsal view.

Distribution: Palaearctic: Russian Far East (Zimin \& Kolomiets 1984. Herting \& DelyDraskovits 1993. Richter 2004). In Korea not mentioned up to the present.

\section{Tachina (Tachina) trigonophora (Zimin, 1980)}

Servillia trigonophora Zimin,1980: 211. Type localities: Korea, Russian Far East: Nature Reserve "Kedrovaya Pad" near Barabash W of Vladivostok.

Distribution: Palaearctic: Russian Far East (Ussuria. Primorie), Korea (Zimin 1980, Ziegler \& Shima 1996, Richter 2004).

\section{Tachina (Tachina) ursina Meigen, 1824}

Tachina ursina Meigen, 1824: 245. Type locality: not given (probably Germany, Stolberg).

Distribution: Palaearctic: Europe northwards to Southern England and Finland. Europian part of Russia, Ukraine, Caucasus. Ural, W and S Siberia. Primorie, S Kuril Islands (Kunashir), Korea. China (Beijing Municipality. Gansu Prov.. Liaoning Prov.. Sichuan Prov.. Shanxi Prov.. Yunnan Prov., Zhejiang Prov.) (Shima 1992, Ziegler \& Shima 1996, Richter 2004. O'Hara. Shima \& Zhang 2009). 


\section{ACKNOWLEDGMENTS}

I wish to thank very much Prof. Hiroshi Shima (Kyushu University Museum, Hakozaki, Fukuoka, Japan) for helpful comments and remarks and the comparison of my description of the female from North Korea with the Japanese D. takanoi Mesnil (terra typica).

I should also like to express my most cordial thanks to Mr Piotr Ślipiński, MSc. Eng., for taking the photographs. My special thanks are due to Mr Tomasz Karolkiewicz for the digital preparation of the figures.

\section{REFERENCES}

ANDERSEN S. 1996. The Siphonini (Diptera, Tachinidae) of Europe. Fauna Entomologica Scandinavica 33:1-146.

BAŃKOWSKA R. \& STERZYŃSKA M. 1997. Faunistic investigations conducted in North Korea by researchers from the Institute of Zoology Polish Academy of Sciences from 1959 to 1990. Fragmenta Faunistica 40: 247-253.

BYSTROWSKI C. 1997. Atylostoma tricolor (Mik, 1883), a species new to the fauna of tachinid flies (Diptera: Tachinidae) of Poland. Fragmenta Faunistica 40: 199-203

CHAo C.- m. 1974. Notes on the Chinese Larvaevoridae. IX. Hystromyia Portschinsky. Acta Entomologica Sinica 17: $474-478$.

CRosskey R. W. 1977. Family Tachinidae. In: DELFTNADO M.D. \& HARDY D. E. (eds), A Catalog of the Diptera of the Oriental Region. Volume III. Suborder Cyclorrhapha (excluding Division Aschiza), pp. 586-697. The University Press of Hawaii. Honolulu, $854 \mathrm{pp}$.

DRABER-MONKO A. 1995. Selected Calyptrata (Diptera) of the pine forests of the Berezinsky Biosphere Reserve in Byelorussia. Fragmenta Faunistica 38: 165-179.

DRABER-MOŃKO A. 2008. State of knowledge of the tachinid fauna of Eastern Asia, with new data from North Korea. Part I. Phasiinae. Fragmenta Faunistica 51: 119-137.

HAN H. Y. \& KIM C. W. 1983. The Korean flies of the Tribe Tachinini (Diptera: Tachinidae). Enomological Research Bulletin 9: 77-94.

HERTING B. \& DELY-DRASKovits Á.1993. Family Tachinidae. Vol.13, pp. 118-458, in Soós Á. Catalogue of Palaearctic Diptera, Anthomyiidae - Tachinidae. Hungarian Natural History Museum, Budapest, 624 pp.

HUA L.-z. 2006. List of Chinese insects. Vol. IV. Sun Yat-sen University Press, Guangzhou. $2+6+540$ pp.

LIM J.- s. \& HAN H.- y. 2008. Redescriptions of two closely resembling Linnaemya species (Insecta: Diptera:Tachinidae) new to Korea. Korean Journal of Systematic Zoology 24: 307-313.

MroczKowsKI M. 1972. Field Investigations in the Democratic People's Republic of Korea by staff members of the Institute of Zoology of the Polish Academy of Sciences. Fragmenta Faunistica18: 313-331.

O'HARA J., SHIMA H. \& ZHANG C. 2009. Annotated Catalogue of the Tachinidae (Insecta: Diptera) of China. Zootaxa 2190: 1-236.

O'HARA J. \& WOOD M. 2004. Catalogue of the Tachinidae (Diptera) of America north of Mexico. Memoires on Entomology, International, 18. iv $+410 \mathrm{pp}$.

RICHTER V. A. 1986. On the fauna of tachinids (Diptera, Tachinidae) of the Far EastUSSR. In: NARTSHUK E. P (ed.), Flies (Diptera) in ecosystems of South Siberia and Far East, USSR Academy of Sciences, Leningrad, $122 \mathrm{pp}$. Proceedings of the Zoological Institute 146: 87-116. [In Russian.],

RICHTER V. A. 1988. New Palaearctic genera and species of tachinids (Diptera, Tachinidae). Systematika Nasekomikh i Kleshchei 70: 202-212. [In Russian.]

RICHTER V. A. 1993. New and little known tachinids (Diptera, Tachinidae) of Transbaikalia and Far East. Èntomologicheskoe Obozrenie, 72: 422-440. [In Russian]

RICHTER V. A. 1999. New and little known tachinids (Diptera, Tachinidae) from the Russian Far East. [In Russian] Ėntomologicheskoe Obozrenie 78: 719-731.

RICHTER V. A. 2004. Fam. Tachinidae - tachinids. In: SIDORENKO V. S., ed., Key to the insects of Russian Far East. Vol. VI. Diptera and Siphonaptera. Part 3, pp. 148-398. Vladivostk, Dal'nauka. 657 pp. [In Russian.]

SHIMA H. 1986. A systematic study of the genus Linnaemya Robineau-Desvoidy from Japan and the Oriental Region (Diptera: Tachinidae). Sieboldia. Acta Biologica 5: 1-96.

SHIMA H. 1992. Tachinidae (Diptera) collected in Ussuri by Prof. T. Saigusa. Makunagi, Acta Dipterologica 17:15-20.

SHIMA H., HAN H. Y. \& TACHI T. 2010. Description of a new genus and six new species of Tachinidae (Diptera) from Asia and New Guinea. Zootaxa 2516: 49-67.

TACHI T. \& SHIMA H. 1998. A systematic study of the genus Actia Robineau-Desvoidy of Japan (Diptera: Tachinidae). Entomological Science 1: 441-463.

TACHI T. \& SHIMA H. 2000. Taxonomic study of the genus Ceromya Robineau-Desvoidy of Japan. Beiträge zur Entomologie. 50: 129-150.

TACHI T. \& SHIMA H. 2002. Systematic study of the genus Peribaea Robineau-Desvoidy of East Asia (Diptera: Tachinidae). Tijdschrift voor Entomologie 145: 115-144. 
TACHI T. \& SHIMA H. 2005. Revision of the subgenus Ceranthia Robineau-Desvoidy of the genus Siphona Meigen of Japan (Diptera: Tachinidae). Entomological Science 8: 189-200.

TACHI T. \& SHIMA H. 2006. Review of genera Entomophaga and Proceromyia (Diptera: Tachionidae). Annals of Entomological Society of America 99: 41-57.

ZIEGLER J. \& SHIMA H. 1996. Tachinid flies of the Ussuri area (Diptera: Tachinidae). Contributions to the knowledge of East Palaearctic insects (5). Beiträge zur Entomologie 46: 379-478.

ZIMIN L. S. 1935. Le systéme de la tribu Tachinini (Diptera, Larvaevoridae). Travaux de l'Institut zoologique de 1'Académie des sciences de 1'URSS 2: 509-636.

ZIMIN L. S. 1980. New species of Tachinids (Diptera, Tachinidae). Èntomologicheskoe Obozrenie 59: $206-222$.

ZIMIN L. S. \& KolomIETS N. G. 1984. Paraziticheskije dvukrylye fauny SSSR (Diptera, Tachinidae). Opredelitel'. Izdatelstvo "Nauka". Sibirskoe Otdelenie. Novosibirsk, 233 pp.

\section{STRESZCZENIE}

[Stan wiedzy o faunie rączyc (Diptera, Tachinidae) Azji Wschodniej, z uwzględnieniem nowych danych z Korei Pólnocnej. Część II. Tachininae]

Opracowanie stanowi kontynuację badań nad fauną rączyc Korei Północnej. Materiał zostal zebrany przez pracowników Instytutu Zoologii PAN w Warszawie w latach 1959-1990 podczas 5 wypraw do Korei Pólnocnej. Muchówki byly zbierane glównie siatką entomologiczną lub czerpakiem, a tylko w dwu ostatnich ekspedycjach zastosowano również żólte pulapki Moerick'a. Podrodzina Tachininae jest jedną $\mathrm{z}$ większych wśród rączyc, w Palearktyce dotychczas stwierdzono około 550 gatunków.

Zgromadzony material Tachininae liczy 90 okazów, jest on jednak dość zróżnicowany. W Korei Pólnocnej w obrębie opracowanej podrodziny znaleziono 29 gatunków z 15 rodzajów. Natomiast z całej Korei (bez podziału na części) w dotychczasowym piśmiennictwie dotyczącym Tachininae wykazano 29 gatunków zaliczanych do 10 rodzajów, w tym z Korei Poludniowej podano dotychczas 12 gatunków z 7 rodzajów Tachininae.

Obecnie z całej Korei znane są 53 gatunki zaliczane do 21 rodzajów, w tym 24 gatunki Tachininae wymienione są z tego kraju po raz pierwszy.

Większość muchówek $\mathrm{z}$ omawianej podrodziny $\mathrm{W}$ stadium larwalnym jest endoparazytoidami glównie gạsienic motyli należących do wielu różnych rodzin. Nieliczne z nich są endoparazytoidami Coleoptera z rodzin Chrysomelidae i Scarabaeidae oraz Hymenoptera z rodzin Vespidae (Eumeninae), Pamphiliidae i Tenthredinidae. Natomiast larwy pojedynczych gatunków rączyc z podrodziny Tachininae rozwijają się jako endoparazy toidy w Chilopoda z rodziny Lithobiidae, Dermaptera z rodziny Forficulidae, Orthoptera z rodziny Tettigoniidae oraz Diptera z rodziny Tipulidae.

W opracowanym materiale wyodrębniono trzy bardzo rzadkie gatunki Tachininae znane jedynie z pierwotnego opisu i nie wykazane dotąd z innych obszarów. Są to: Nigara gracilis Richter, Peleteria pallida Zimin i Tachina (Tachina) majae Zimin.

W wykazie systematycznym rączyc przy trzech gatunkach stwierdzonych w Korei Pólnocnej zamieszczono uwagi dotyczace ich morfologii. Sa to: Dolichopodomintho takanoi Mesnil, Hystriomyia nigrosetosa Zimin i Nigara gracilis Richter.

W pracy kolorowymi fotografiami zilustrowano dziesięć rzadko wykazywanych gatunków rączyc - nowych dla fauny Korei. 